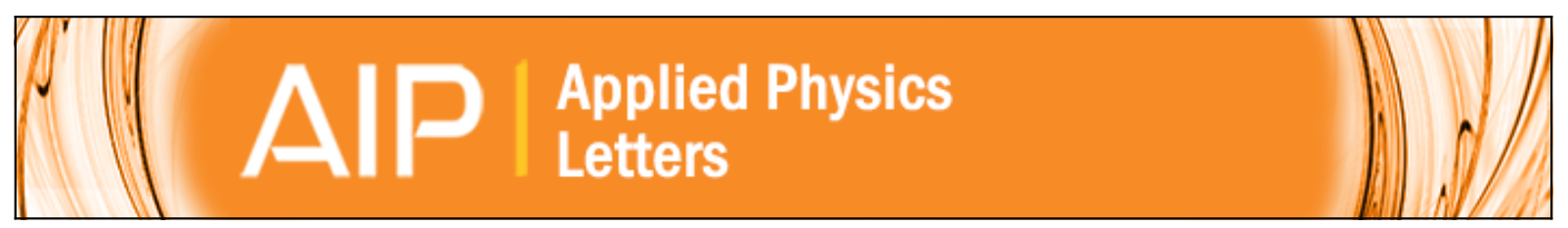

\title{
A detailed study of magnetization reversal in individual Ni nanowires
}

Enrique Vilanova Vidal, Yurii P. Ivanov, Hanan Mohammed, and Jürgen Kosel

Citation: Applied Physics Letters 106, 032403 (2015); doi: 10.1063/1.4906108

View online: http://dx.doi.org/10.1063/1.4906108

View Table of Contents: http://scitation.aip.org/content/aip/journal/apl/106/3?ver=pdfcov

Published by the AIP Publishing

\section{Articles you may be interested in}

Kondorski reversal in magnetic nanowires

J. Appl. Phys. 115, 17D137 (2014); 10.1063/1.4865975

Observation of magnetocapacitance in ferromagnetic nanowires

Appl. Phys. Lett. 101, 052401 (2012); 10.1063/1.4739848

Magnetic reversal of cylindrical nickel nanowires with modulated diameters

J. Appl. Phys. 109, 033907 (2011); 10.1063/1.3544036

Field and current-induced magnetization reversal studied through spatially resolved point-contacts

J. Appl. Phys. 107, 103909 (2010); 10.1063/1.3407539

Magnetic properties of arrays of nanowires: Anisotropy, interactions, and reversal modes

J. Appl. Phys. 107, 09B504 (2010); 10.1063/1.3350905

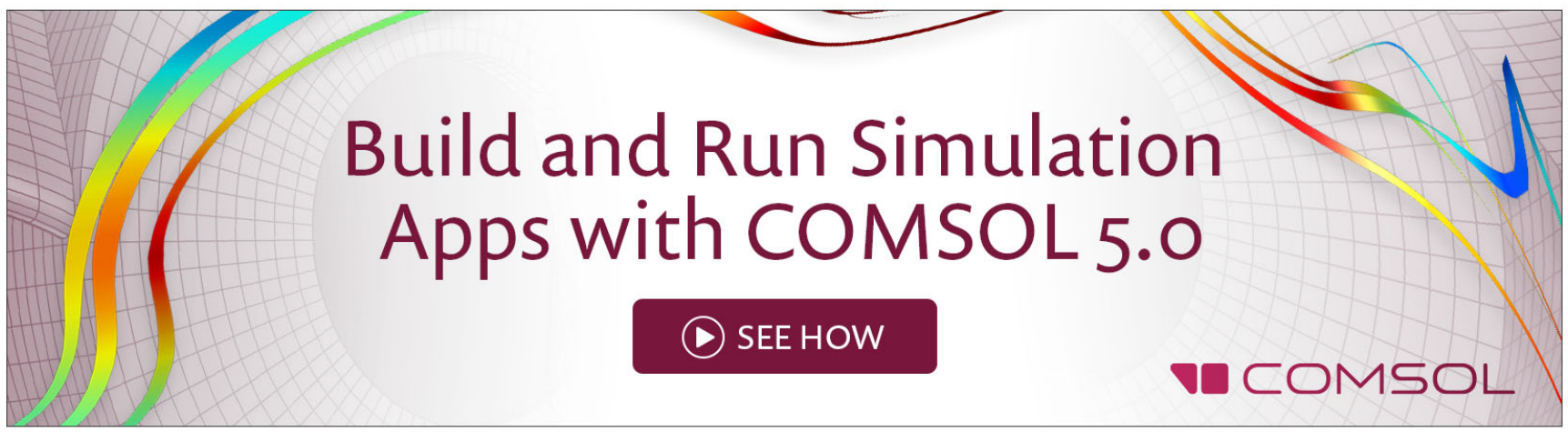




\title{
A detailed study of magnetization reversal in individual Ni nanowires
}

\author{
Enrique Vilanova Vidal, Yurii P. Ivanov, Hanan Mohammed, and Jürgen Kosel \\ Division of Computer, Electrical and Mathematical Sciences and Engineering, King Abdullah University \\ of Science and Technology, Thuwal 23955, Kingdom of Saudi Arabia
}

(Received 26 October 2014; accepted 6 January 2015; published online 20 January 2015)

\begin{abstract}
Magnetic nanowires have emerged as essential components for a broad range of applications. In many cases, a key property of these components is the switching field, which is studied as a function of the angle between the field and the nanowire. We found remarkable differences of up to $100 \%$ between the switching fields of different nanowires from the same fabrication batch. Our experimental results and micromagnetic simulations indicate that the nanowires exhibit a single domain behavior and that the switching mechanism includes vortex domain wall motion across the nanowire. The differences between the switching fields are attributed to different cross-sections of the nanowires, as found by electron microscopy. While a circular cross-section yields the smallest switching field values, any deviation from this shape results in an increase of the switching field. The shape of the nanowires' cross-sections is thus a critical parameter that has not been previously taken into account. C 2015 AIP Publishing LLC. [http://dx.doi.org/10.1063/1.4906108]
\end{abstract}

The interest in nanowire (NW)-based systems has increased considerably during the last decade, because of their potential applications ranging from the treatment of diseases ${ }^{1-3}$ to microfluidics, ${ }^{4}$ and spintronics devices. ${ }^{5,6} \mathrm{NWs}$ fabricated inside of anodic aluminum oxide (AAO) templates are especially attractive because they offer several advantages, such as high packing density and highly ordered distributions. ${ }^{7}$ In addition, they grow inside an isolating template and perpendicular to the substrate. This feature, applied to magnetic memory devices, like race track memories, ${ }^{8}$ would allow the data storage density to be increased by creating 3D storage media. In this context, it is crucial to have a complete understanding of the magnetic behavior of individual NWs, because any memory device will be composed of multiple individual NWs.

The objective of this paper is to offer a comprehensive view of the reversal process in individual NWs and of the role played by the NWs' cross-section shape, which is usually neglected.

Classically, the Stoner-Wohlfarth and Kondorsky models are utilized to understand the basic magnetic behavior of a NW. Both cannot take into account some of the peculiar properties of NWs or specific effects of cross-section shapes. On the other hand, the micromagnetic simulations performed here are able to reproduce the experimental results, as will be shown later.

The distinction between $\mathrm{H}_{\mathrm{c}}$ and $\mathrm{H}_{\mathrm{sw}}$ should first be clarified. $\mathrm{H}_{\mathrm{c}}$ refers to the magnetic field value at which the average magnetization of a single $\mathrm{NW}$ is equal to zero. $\mathrm{H}_{\mathrm{sw}}$ is the magnetic field value at which the domain wall propagation in a single NW is triggered, which eventually leads to a reversal of the magnetization.

A priori, the magnetic easy axis of a Ni NW, with $160 \mathrm{~nm}$ in diameter and fcc structure, is expected to be determined by the NW's shape, because it possesses a very small magneto crystalline anisotropy constant $\left(K_{a}=-0.048 \times 10^{-5}\right){ }^{9}$ At remanence, this will cause the magnetic moments to be parallel to the NW's axis. Exceptions are the NW ends, where open vortex areas (OVA) appear ${ }^{10,11}$ to minimize the magnetostatic energy.

For Ni NWs with diameters greater than $50 \mathrm{~nm}$, a vortex domain wall (VDW) reversal mode is expected. ${ }^{10,12}$

Porous AAO membranes are fabricated from highly pure $(99.9995 \%)$ Aluminum discs, with $25 \mathrm{~mm}$ in diameter and $0.5 \mathrm{~mm}$ in thickness, using a two-step anodization process. Details of the fabrication process can be found elsewhere. ${ }^{7}$ Briefly, the fabrication of the AAO membrane is completed by an exponential reduction of the potential to create conducting paths (dendrites) through a thin layer of Alumina between the pores and the Aluminum layer at the bottom of the discs. Ni NWs are grown inside the pores of the AAO membrane by pulsed electrodeposition. In all experiments, the NWs are dispersed on a silicon substrate with a silicon oxide layer on top of it.

Based on their morphology, NWs produced by this method can be divided into two parts: the main part, which in the ideal case has a cylindrical shape; and the dendrites, which have a root-like shape (Figure 1(a)).

To characterize the NWs' crystal structure and their morphology, we used a Titan CT transmission electron microscope (TEM) from FEI and a Quanta 3D scanning electron microscope (SEM) from FEI.

Figure 1(a) shows an example of a dendrite area. It typically consists of two to four dendrites, and their shape slightly differs from wire to wire. Its total length is roughly the same for all NWs and approximately $500 \mathrm{~nm}$. The TEM image in Figure 1(b) shows a cross-section of a NW. This "real" cross-section can deviate considerably from a circular shape, which would be expected from an "ideal" cylindrical NW. It is the result of non-circular pore shapes, which are commonly observed in AAO membranes. ${ }^{13,14}$ The NWs have a smooth surface and polycrystalline $\mathrm{Ni}$ fcc structure (Figure 1(d)).

Single NW magnetic characterization is performed on $12 \mu \mathrm{m}$ long NWs via the magneto-optical Kerr effect (MOKE) and Magnetic Force Microscopy (MFM) using a 

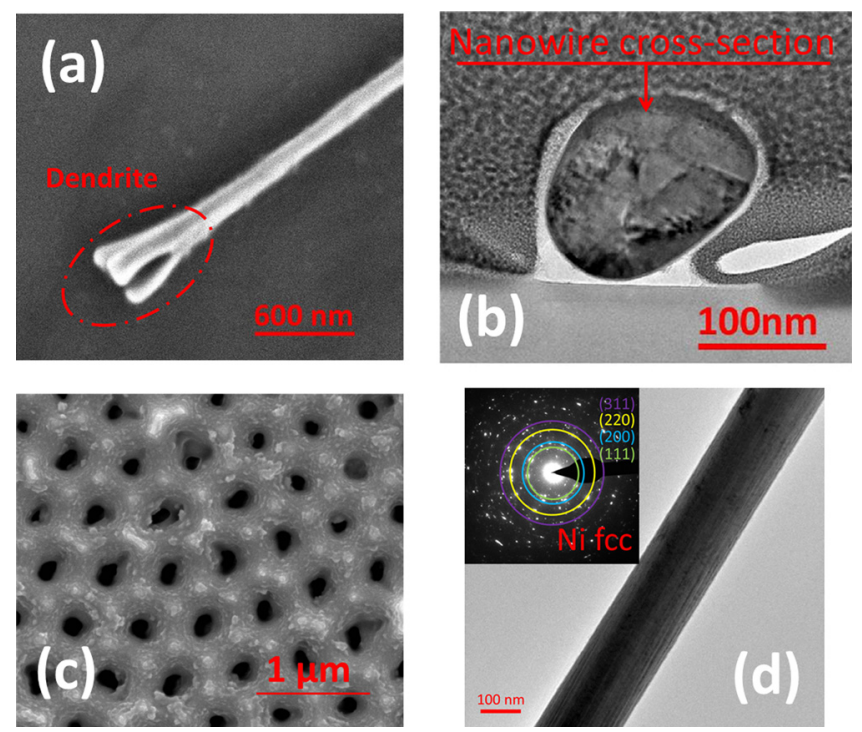

FIG. 1. (a) and (c) SEM images showing: (a) A NW with dendrites; (c) An AAO membrane coated with $6 \mathrm{~nm}$, of platinum. (b) and (d) TEM images of NWs showing: (b) a typical cross-section; (d) the diffraction pattern (inset) of a NW section.

NanoMOKE $2 \circledR$ from Quantum Design and a MFP-3D-Bio from Asylum Research, respectively. Each MOKE loop recorded is the result of 200 averaged loops. $\mathrm{H}_{\mathrm{sw}}$ is extracted by calculating the maximum of the first derivative of the raw data. For the MFM measurement, a magnetic field is applied to saturate the NW in a particular direction. Afterwards, the magnetic field is removed, and the NW is imaged at remanence. Then, the magnetic field is increased in steps of $40 \mathrm{Oe}$ in the opposite direction and removed to carry out subsequent MFM measurements. The magnetic field value for which a change in contrast at the ends of the NW is observed is considered as $\mathrm{H}_{\mathrm{sw}}$. Each MFM scan has been performed at remanence (zero applied field), in order to avoid effects of the stray field of the MFM tip on the NW's switching field. The resolution of those two methods enabled the measurement of $\mathrm{H}_{\mathrm{sw}}$ up to $70^{\circ}$.

As shown in Figure 2(a), $\mathrm{H}_{\mathrm{sw}}$ increases with increasing values of $\theta$. Interestingly, the switching field of the NWs
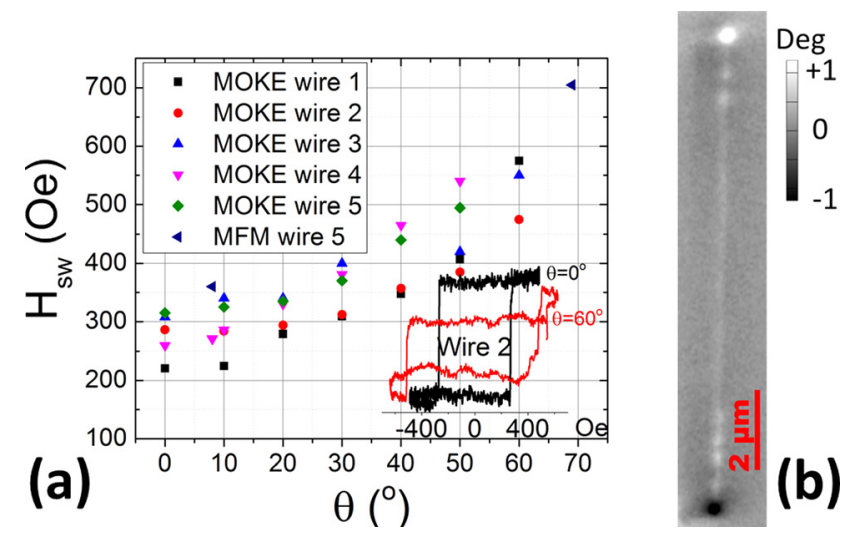

FIG. 2. (a) The angular dependence of the switching field $\left(\mathrm{H}_{\mathrm{sw}}\right)$ of five single NWs measured by MOKE. The inset shows examples of hysteresis loops measured by MOKE at $\theta=0^{\circ}$ and $\theta=60^{\circ}$ (wire 2). Note, the saturation values are different, since only the longitudinal component is captured and it decreases as $\theta$ is increased. (b) An MFM image of a NW at remanence. differs from NW to NW. The difference in $\mathrm{H}_{\mathrm{sw}}$ between NW 1 and 5 is approximately $100 \%$ at $\theta=0^{\circ}$, and this difference remains roughly constant over all angles. In the case of $\mathrm{NW}$ 1 and 2 , the difference is roughly $100 \%$ at $\theta=0^{\circ}$, but upon increasing $\theta$, the difference reduces and even inverts. The results show that different NWs can even exhibit different angular dependences. The inset in Figure 2(a) shows typical hysteresis loops recorded by MOKE at $\theta=0^{\circ}$ and $\theta=60^{\circ}$. Sharp transitions between magnetic states indicate that there is a single magnetic domain behavior. The MFM image of a NW at remanence in Figure 2(b) confirms this result, showing the typical contrast of a single magnetic domain NW. The weak variation of the MFM contrast in Figure 2(b) is caused by non-magnetic contributions from surface features, which are usually more accentuated near the ends of the wires.

The influence of dendrites on the magnetization behavior of NWs has not been investigated before. However, dendrites might be considered as shape defects, which could significantly change the value of $\mathrm{H}_{\mathrm{sw}}$. To study the influence of the dendrites, we measure $\mathrm{H}_{\mathrm{sw}}$ as a function of $\theta$ in one NW with and without dendrites. For this purpose, approximately $1 \mu \mathrm{m}$ of the NW including the dendrites is removed using a focused ion beam. The effect of this length reduction on $\mathrm{H}_{\mathrm{sw}}$ can be neglected, since the length/diameter ratio is still larger than 100.

A comparison of the dependence of $\mathrm{H}_{\mathrm{sw}}$ on $\theta$ of the NW before and after removing the dendrites indicates that the dendrites do not have a measurable effect on $\mathrm{H}_{\mathrm{sw}}$ (Figure 3). As a possible explanation, we suggest that dendrites behave as pinning sites for domain wall propagation. In a NW with a dendrite, the switching process is triggered from the end opposite of the dendrite. Once the dendrite is removed, the switching can be triggered at either end at similar values of $\mathrm{H}_{\mathrm{sw}}$.

To comprehend the source of the scattering obtained for $\mathrm{H}_{\mathrm{sw}}$ of different NWs (Figure 2) and to study the switching
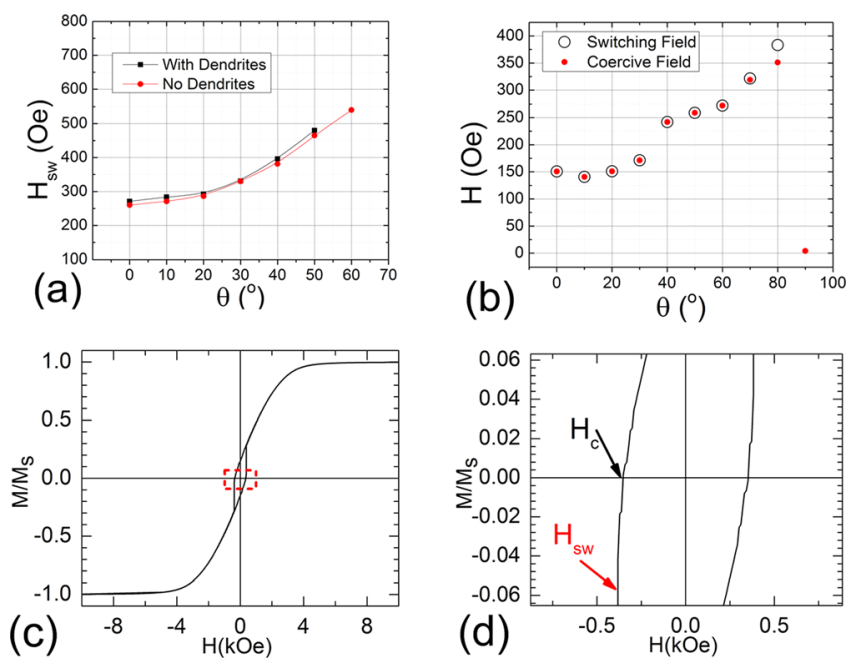

FIG. 3. (a) The switching field $\left(\mathrm{H}_{\mathrm{sw}}\right)$ of a single $\mathrm{NW}$ measured by MOKE at different angles $(\theta)$ between the magnetic field and the NW axis, before and after removing the dendrites. (b) Simulated $\mathrm{H}_{\mathrm{c}}$ and $\mathrm{H}_{\mathrm{sw}}$ for a single nanowire (with circular cross-section) at different angles $(\theta)$. (c) Simulated hysteresis loop at $\theta=80^{\circ}$. (d) Zoom of the squared area in Figure 3(c). 
field at $\theta>70^{\circ}$, we run micromagnetic simulations based on the Landau-Lifsthitz-Gilbert (LLG) equation. ${ }^{15}$

We simulated the magnetization reversal process and its angular dependence using the Magpar package with finite element discretization ${ }^{16,17}$ with typical magnetic parameters of bulk fcc Ni. ${ }^{12}$ To emulate the polycrystalline structure of real NWs, a mesh of tetrahedra is randomly assigned. The diameter of the simulated NWs is $160 \mathrm{~nm}$, and the average size of the mesh is $6 \mathrm{~nm}$, which is approximately the exchange length of $\mathrm{Ni}^{12}$ The simulations are performed utilizing a circular and a real cross-section (Figure 1(b)).

To reduce the computational requirements, the simulated NW length is $3 \mu \mathrm{m}$ instead of the original length of $12 \mu \mathrm{m}$. This yields only a minor reduction in the shape anisotropy by $4 \%$ (see the detailed discussion in the supplementary material). ${ }^{18}$

The simulations offer a means to visualize the magnetic moment distribution (examples provided in supplementary material $)^{18}$ and hysteresis loops. From the maximum value of the first derivative of the simulated loops (Figure 3(c)), we determine the $\mathrm{H}_{\mathrm{sw}}$ values (Figures 3(b) and 4), while $\mathrm{H}_{\mathrm{c}}$ is obtained from the condition $\mathrm{M}=0$.

At $\theta<70^{\circ}$, the component of the applied magnetic field, which is parallel to the NW axis, reverses the magnetization via nucleation of VDWs at the NW ends, which propagate toward the opposite sides. Nucleation of the VDW occurs before the magnetization becomes zero; therefore, $\mathrm{H}_{\mathrm{sw}}$ and $\mathrm{H}_{\mathrm{c}}$ have the same values.

At $70^{\circ}<\theta<90^{\circ}$ (Figure 3), the rotation of the magnetic moments, due to the perpendicular component of the magnetic field, results in the average magnetic moment (parallel to the applied field) being equal to zero at $\mathrm{H}=\mathrm{H}_{c}$, (Figure 3(d)). However, the magnetization is still not reversed. Upon further increase of the magnetic field, the longitudinal component causes VDW nucleation and propagation (reversing the magnetic moments). Because of this, different values are obtained for $\mathrm{H}_{\mathrm{c}}$ and $\mathrm{H}_{\mathrm{sw}}$ (Figure 3(d)), a feature that is not predicted by the Kondorsky model.

At $\theta=90^{\circ}$, the magnetic moments reverse via quasicoherent rotation, and nucleation of a VDW is not observed.

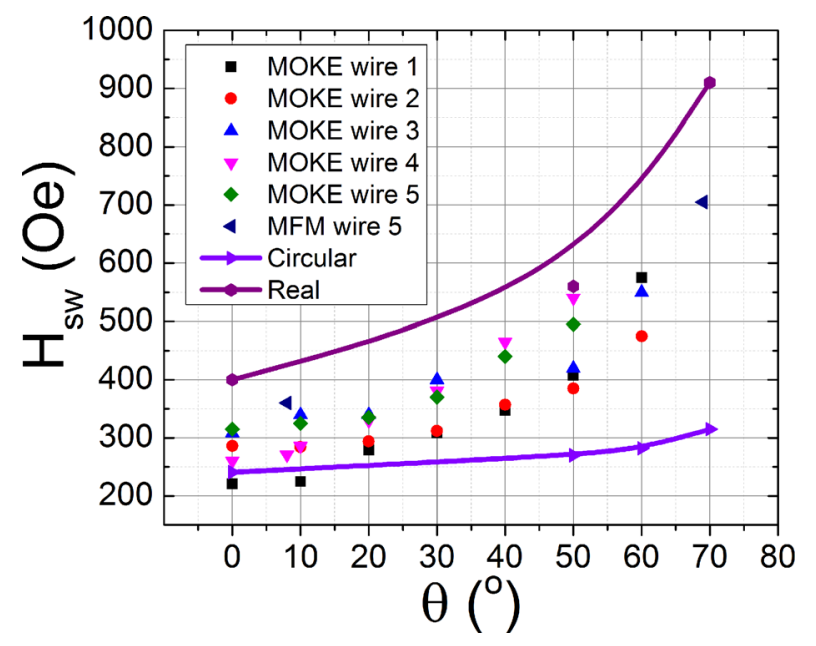

FIG. 4. Switching field $\left(\mathrm{H}_{\mathrm{sw}}\right)$ values obtained by MOKE and MFM measurements (dots) and by simulations (dots joined by lines) at different angles $(\theta)$ between the magnetic field and the NW axis.
The concept of a switching field is, therefore, no longer applicable.

As can be seen in Figure 4, the values of $\mathrm{H}_{\mathrm{sw}}$ calculated for the two different cross-section shapes are remarkably different. For $\theta=0^{\circ}$, the value for a NW with a circular crosssection is $241 \mathrm{Oe}$ and the one for the real cross-section is $400 \mathrm{Oe}$. In addition, the two curves of the angular dependences have different slopes, meaning that at larger values of $\theta$, the differences increase. The $\mathrm{H}_{\mathrm{sw}}$ values obtained from the circular and real cross-sections define a range inside which all of our experimental data fit. This suggests that the different $\mathrm{H}_{\mathrm{sw}}$ values found for different NWs are a result of their slightly different cross-sectional shapes. While the simulation with the circular cross-section yields the smallest possible values for $\mathrm{H}_{\mathrm{sw}}$, it might be possible to observe even larger values than the ones found for the real cross-section, depending on the actual cross-section shape of the NW ends.

The simulation also shows some interesting features of the OVA. The chirality of the OVA is not defined a priori. Thus, once the magnetic field is removed after saturating the NW, the OVAs can have either the same or opposite chirality (Figures 5(a) and 5(b)).

Another feature predicted by the simulation is the increase in the OVA's length by $\mathrm{d} \approx 50 \mathrm{~nm}$ (Figures 5(a) and $5(b))$. This feature is found at high values of $\theta$ and results from the contribution of the perpendicular magnetic field component.

Figure 6 shows the positions of the OVA cores at the NW ends and how these positions shift depending on $\theta$ and the chirality of the OVA. This core shift becomes more pronounced at higher angles. At $\theta=0^{\circ}$, there is no transversal component of the applied field that could cause a shift (Figure 5(a)).

If the OVAs have the same chirality, their cores are displaced in the same direction at $\theta$ greater than $0^{\circ}$. If the OVAs have a different chirality, their cores are displaced in the opposite direction. This last case is depicted in Figure 6(b).

In this study, we analyzed the angular dependence of $\mathrm{H}_{\mathrm{sw}}$ and $\mathrm{H}_{\mathrm{c}}$ of single Ni NWs. Both increased with increasing $\theta$ and shared the same value up to $\theta=70^{\circ}$ beyond which $\mathrm{H}_{\mathrm{sw}}>\mathrm{H}_{\mathrm{c}}$. We observed a single magnetic domain state by MOKE and MFM, which was confirmed by simulations. The simulations also show the magnetic reversal process, which occurs by vortex nucleation followed by domain wall propagation along the wire. The VDW chirality is determined by the chirality of the open vortex found at the NW's ends, which is the result of a random process.

The simulations also indicate a dependence of the size of the OVA at the ends of the wires on $\theta$. The OVA becomes

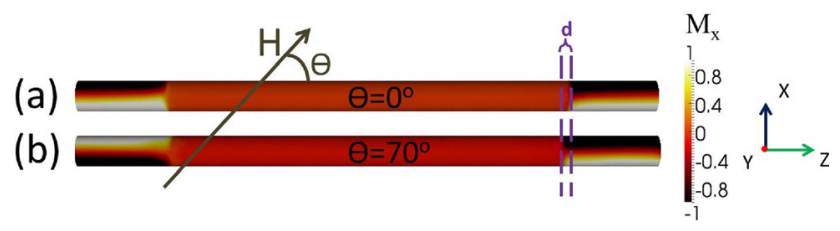

FIG. 5. Magnetization of two individual NWs just before switching occurs. (a) $\theta=0^{\circ}$. (b) $\theta=70^{\circ}$. Dashed lines indicate the increase in the length of the OVA. 
(a)

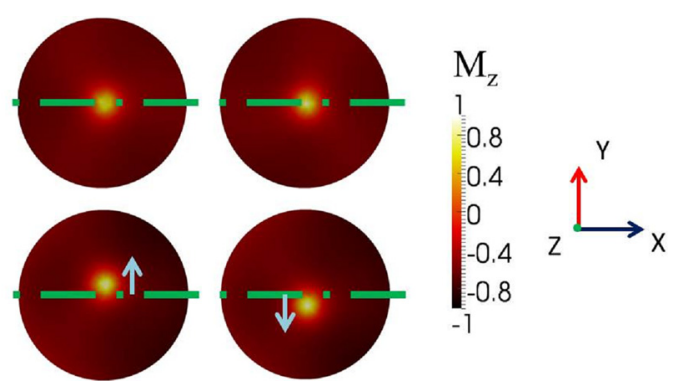

FIG. 6. Side views of NWs from Figure 5. (a) Applied field $\theta=0^{\circ}$. (b) Applied field $\theta=70^{\circ}$ and OVAs have different chirality. The green dashed lines indicate the center lines of the NWs. The blue arrows indicate the OVA core displacement.

more elongated as $\theta$ increases. Due to the transverse component of the applied magnetic field at $\theta>0^{\circ}$, the core of the OVA shifts from the central position and the direction of shifting is determined by the chirality.

The experimental results of the angular dependence show that the presence of dendrites does not have measurable effects. This means that a fabrication process that avoids the formation of dendrites might not be necessary in device production.

The shape of the cross-section of the NW strongly influences $\mathrm{H}_{\mathrm{sw}}$ and can change its value by more than $100 \%$. This is an important observation that has been neglected in previous studies. It is particularly relevant from a technological point of view, where the properties of individual NW's need to be taken into account for device design.

The research reported in this publication was supported by King Abdullah University of Science and Technology
(KAUST). We would also like to thank the financial support provided by SABIC.

${ }^{1}$ A. O. Fung, V. Kapadia, E. Pierstorff, D. Ho, and Y. Chen, J. Phys. Chem. C 112(39), 15085 (2008).

${ }^{2}$ D. S. Choi, J. Park, S. Kim, D. H. Gracias, M. K. Cho, Y. K. Kim, A. Fung, S. E. Lee, Y. Chen, S. Khanal, S. Baral, and J. H. Kim, J. Nanosci. Nanotechnol. 8(5), 2323 (2008).

${ }^{3}$ D. S. Choi, X. Hopkins, R. Kringel, J. Park, I. T. Jeon, and Y. K. Kim, J. Appl. Phys. 111, 07B329 (2012).

${ }^{4}$ A. Alfadhel, B. Li, A. O. Zaher, O. Yassine, and J. Kosel, Lab Chip 14, 4362 (2014).

${ }^{5}$ M. Yan, A. Kákay, S. Gliga, and R. Hertel, Phys. Rev. Lett. 104, 057201 (2010).

${ }^{6}$ R. Wieser, E. Y. Vedmedenko, P. Weinberger, and R. Wiesendanger, Phys. Rev. B 82, 144430 (2010).

${ }^{7}$ A. Eftekhari, Nanostructured Materials in Elctrochemistry (Wiley, 2008).

${ }^{8}$ S. S. P. Parkin, M. Hayashi, and L. Thomas, Science 320(5873), 190 (2008).

${ }^{9}$ Y. Liu, D. J. Sellmyer, and D. Shindo, Handbook of Advanced Magnetic Materials, Nanostructural Effects vol. 1 (Springer, Berlin, 2005).

${ }^{10}$ R. Hertel, J. Magn. Magn. Mater. 249(1-2), 251 (2002).

${ }^{11}$ T. Wang, Y. Wang, Y. Fu, T. Hasegawa, F. S. Li, H. Saito, and S. Ishio, Nanotechnology 20, 105707 (2009).

${ }^{12}$ Yu. P. Ivanov, M. Vázquez, and O. Chubykalo-Fesenko, J. Phys. D: Appl. Phys. 46(48), 485001 (2013).

${ }^{13}$ Ch. Kuo and Ch. Chen, Mater. Trans. 50(5), 1102 (2009).

${ }^{14}$ L. Zaraska, G. D. Sulka, and M. Jaskula, J. Phys.: Conf. Ser. 146, 012020 (2009).

${ }^{15}$ L. G. Vivas, Yu. P. Ivanov, D. G. Trabada, M. P. Proenca, O. ChubykaloFesenko, and M. Vázquez, Nanotechnology 24, 105703 (2013).

${ }^{16}$ Yu. P. Ivanov, L. G. Vivas, A. Asenjo, A. Chuvilin, O. ChubykaloFesenko, and M. Vazquez, Europhys. Lett. 102, 17009 (2013).

${ }^{17}$ W. Scholz, J. Fidler, T. Schrefl, D. Suess, R. Dittrich, H. Forster, and V. Tsiantos, Comput. Mater. Sci. 28, 366 (2003).

${ }^{18}$ See supplementary material at http://dx.doi.org/10.1063/1.4906108 for simulation consideration and magnetic moment reversing process visualization. 